\title{
Improved Malaria Case Management after Integrated Team-based Training of Health Care Workers in Uganda
}

\author{
Umaru Ssekabira, Hasifa Bukirwa, Heidi Hopkins, Allen Namagembe, Marcia R. Weaver, Lydia Mpanga Sebuyira, \\ Linda Quick, Sarah Staedke, Adoke Yeka, Moses Kiggundu, Gisela Schneider, Keith McAdam, \\ Fred Wabwire-Mangen, and Grant Dorsey* \\ Joint Uganda Malaria Training Program, Kampala, Uganda; Uganda Malaria Surveillance Project, Kampala, Uganda; Department of \\ Medicine, San Francisco General Hospital, University of California, San Francisco, California; Department of Health Services and \\ International Training and Education Center on HIV (I-TECH), Seattle, Washington; Infectious Diseases Institute, and School of \\ Public Health, Makerere University, Kampala, Uganda; Centers for Disease Control and Prevention, Atlanta, Georgia; London \\ School of Hygiene and Tropical Medicine, London, United Kingdom
}

\begin{abstract}
Malaria case management in Africa is characterized by presumptive treatment and substantial overtreatment. We evaluated an integrated team-based training program on malaria case management. Surveillance data 120 days before and after training were compared at eight health facilities in Uganda. After training, the proportion of patients with suspected malaria referred for blood smears increased from $38.3 \%$ to $54.6 \%(P=0.04)$ in persons $<5$ years of age years and from $34.1 \%$ to $53.4 \%(P=0.02)$ in those $\geq 5$ years of age. The proportion of patients with negative blood smears prescribed antimalarial drugs decreased from $47.9 \%$ to $19.6 \%(P<0.001)$ in persons $<5$ years of age and from $38.8 \%$ to $15.6 \%(P<0.001)$ in those $\geq 5$ years of age. Training did not improve the proportion of patients with positive blood smears prescribed antimalarial drugs, the proportion of patients prescribed appropriate antimalarial drugs, or the diagnostic accuracy of microscopy. Integrated team-based training may improve malaria case management and reduce the number of unnecessary antimalarial treatments.
\end{abstract}

\section{INTRODUCTION}

In Uganda and other parts of Africa, presumptive treatment of malaria is common practice. ${ }^{1,2}$ In many facilities, supplies and staff for good-quality laboratory diagnosis are not available. Even where diagnostic capabilities exist, studies have found that health workers often do not request a diagnostic test for patients suspected to have malaria., ${ }^{3,4}$ When tests are requested, health workers often ignore the results, prescribing antimalarial drug treatment despite a negative test result. $^{2-5}$ In addition, when antimalarial treatment is prescribed, the prescription is not always for an appropriate regimen. ${ }^{6}$

Presumptive treatment was accepted and even encouraged in official guidelines when older, inexpensive antimalarial drugs such as chloroquine were widely effective. In addition, the anti-inflammatory properties of chloroquine may effectively manage other non-malarial febrile conditions. However, in many settings, empiric treatment results in substantial overuse of antimalarial drugs and delays in the diagnosis of other febrile illnesses. ${ }^{2,7-9}$ For example, bacteremia is an important, treatable cause of fever and coma resembling malaria. ${ }^{10}$ Inappropriate use of antimalarial drugs is increasingly problematic because older antimalarial drugs are replaced by new artemisinin-based combination therapies (ACTs) that are more costly, in limited supply, and have less established safety records. ${ }^{11,12}$ The need for improved malaria case management in sub-Saharan Africa is recognized as a public health issue requiring urgent attention. ${ }^{13-15}$

Most countries in sub-Saharan Africa have recently changed their antimalarial drug treatment policies, adopting ACTs as the recommended treatment of uncomplicated malaria. The widespread implementation of these new highly effective drugs provides an important opportunity to substantially improve the treatment of malaria. However, it is im-

\footnotetext{
* Address correspondence to Grant Dorsey, University of California, San Francisco, Box 0811, San Francisco, CA 94143. E-mail: gdorsey@medsfgh.ucsf.edu
}

perative that these drugs are used rationally to maximize their impact. Training of health workers and improving diagnostic capabilities have been identified as potential avenues for improving malaria case management. The results of observational studies suggest a need for more integrated training approaches ${ }^{6}$ and supportive supervision. ${ }^{16}$ However, recent intervention studies highlight the challenges faced in changing in health workers' diagnostic and prescribing practices. ${ }^{5}$

In response to concerns about the need for improved case management in Uganda, we designed and prospectively evaluated a training curriculum for health workers at facilities with existing laboratory services. The Malaria Control Program (MCP) of the Uganda Ministry of Health recommends diagnostic testing for malaria at all sites with a functional laboratory. We evaluated the impact of the training on the quality of case management in eight health facilities approximately one year after the implementation of artemetherlumefantrine as the recommended first-line treatment for uncomplicated malaria. Outcomes were measured using an existing targeted surveillance system that captures data on outpatient demographics, diagnoses, and treatments prescribed.

\section{METHODS}

Study sites and population. The training intervention was implemented at eight sentinel sites that were established in 1998 by the Uganda Ministry of Health. Sites were selected to represent the diversity of geography and malaria transmission intensity in Uganda (Table 1). All sites are government-run health centers IV, with the exception of Kabale, which is a regional referral hospital, and all have a functional laboratory with microscopy services. At the time of this study, rapid diagnostic tests were not in use at any of the sites. Health centers IV have a catchment population of approximately 100,000 people and are typically staffed by one medical officer, two clinical officers, five nurses, five midwives, four nursing assistants, one dental officer, one laboratory technician, one laboratory assistant, one records officer, one health educator, and one health assistant. Kabale regional referral 
TABLE 1

Characteristics of study sites and patient encounters, Uganda*

\begin{tabular}{|c|c|c|c|c|c|c|c|c|c|}
\hline \multirow[b]{3}{*}{ Entomologic inoculation rate ${ }^{17,18}$} & \multicolumn{9}{|c|}{ Study site } \\
\hline & Kabale & Kamwezi & Kihihi & Walukuba & Kyenjojo & Omugo & Nagongera & Aduku & Total \\
\hline & $<1$ & $\mathrm{NA}$ & 6 & 6 & 7 & 397 & 562 & 1586 & \\
\hline Last date of training & $12 / 2 / 2006$ & $12 / 20 / 2006$ & $12 / 20 / 2006$ & 6/9/2007 & $3 / 31 / 2007$ & $2 / 14 / 2007$ & 6/9/2007 & 2/14/2007 & \\
\hline \multicolumn{10}{|c|}{ Characteristics of patient encounters } \\
\hline \multicolumn{10}{|l|}{ No. patient encounters } \\
\hline Pre-training & 9,672 & 5.885 & 9,651 & 9,091 & 6,246 & 6,479 & 7,402 & 4,922 & 59,348 \\
\hline Post-training & 14,060 & 8,760 & 9,948 & 8,772 & 6,268 & 7,515 & 6,868 & 5,514 & 67,705 \\
\hline \multicolumn{10}{|l|}{ Age $<5$ years } \\
\hline Pre-training, no. (\%) & $1,476(15)$ & $870(15)$ & $3,218(33)$ & $2,385(26)$ & $1,232(20)$ & $1,877(29)$ & $2,338(32)$ & $1,397(28)$ & $14,793(25)$ \\
\hline Post-training, no. (\%) & $2,326(17)$ & $1,478(17)$ & $3,270(33)$ & $2,072(24)$ & $1,138(18)$ & $2,464(33)$ & $2,112(31)$ & $1,648(30)$ & $16,508(24)$ \\
\hline \multicolumn{10}{|l|}{ Blood smear positive $\dagger$} \\
\hline Pre-training, no. $(\%)$ & $229(21)$ & $225(39)$ & $1,491(34)$ & $1,233(54)$ & $391(35)$ & $449(43)$ & $287(33)$ & $320(62)$ & $9,870(47)$ \\
\hline Post-training, no, (\%) & $290(12)$ & $90(12)$ & $1,770(33)$ & $2,283(57)$ & $650(35)$ & $257(33)$ & $373(39)$ & $575(54)$ & $4,323(31)$ \\
\hline
\end{tabular}

$* \mathrm{NA}=$ not available.
$\dagger$ Denominator includes those in whom a blood smear was obtained

hospital has a catchment population of approximately two million persons and a larger staff. Health care services are provided free of charge at all centers.

Malaria surveillance system. The Uganda Malaria Surveillance Project (UMSP) and the MCP established an on-going malaria surveillance system at the selected sentinel sites beginning in 2006. The system strengthens data collection by providing standardized registers to record patient demographics, laboratory results, diagnoses, and treatments prescribed for all patients presenting to the sentinel sites. The UMSP staff visit the health facilities on a monthly basis for supervision, collection of data, and provision of feedback to staff about data collected in previous months.

Training intervention. The aim of the training was to improve health workers' performance of clinical and laboratory tasks relevant to malaria case management, and to encourage a shared understanding of the management approach and the role of each staff member. The curriculum and training materials were developed and delivered through the Joint Uganda Malaria Training Program (JUMP), a partnership between UMSP and the Infectious Diseases Institute (IDI) of Makerere University. The course was team-based and targeted three categories of staff typically working in health facilities in Uganda: clinicians (medical officers, clinical officers, nurses, and midwives), laboratory staff, and records clerks. The course includes both didactic and practical hands-on sessions. Training modules include information on malaria transmission and epidemiology, Uganda malaria policy, medical ethics, clinical management of malaria, preparation of blood smears, microscopy skills, and medical record keeping. The modules on clinical management of malaria included a session on management of patients with fever but with a negative blood slide for malaria parasites. Details are available at http://www.idi.ac.ug/resources/JUMPCurriculum.pdf and http://www.muucsf.org/publications/Training_materials.asp).

To ensure consensus among key stakeholders and cohesion with national case management guidelines, the training materials were developed through an iterative process with input from many sources. Existing resource materials and guidelines issued by the Uganda Ministry of Health, the World Health Organization, including the Integrated Management of Childhood Illness (IMCI) strategy, and other training and public health institutions were reviewed. A curriculum development specialist led the process in which training modules were written by experts in malaria epidemiology, laboratory diagnosis, and antimalarial treatment, and were edited by colleagues with field and training experience. Two national stakeholders' meetings were held to formally review and revise subsequent curriculum drafts, informed by pilot trainings of 50 health workers. The final curriculum and training materials were approved by Uganda's National Malaria Case Management Technical Working Group, the MCP, and other stakeholders in Uganda.

Each site was visited by the JUMP team prior to training as part of a baseline needs assessment. A total of 170 health workers received training from the 8 sentinel site health facilities from December 2006 through June 2007 (Table 1). Training consisted of a six-day course conducted at the IDI and UMSP core facilities at Mulago Hospital in Kampala. To minimize disruption of patient services, the staff at each health facility was divided into two groups and trained in two back-to-back week-long sessions. Trainees were assessed for learning attainment in terms of knowledge, skills, and attitudes through performance on written pre-test and post-test, participation in clinical and case discussions, and attendance of at least $90 \%$ of the sessions. Two follow-up support supervision visits approximately 6 and 12 weeks after the initial training course were conducted at the sentinel sites by JUMP team members to reinforce training messages, assess skills, and provide individual feedback.

Microscopy and quality control. We compared the diagnostic accuracy of field microscopy before and after training using expert microscopy as the gold standard. Thick blood smears were collected from 1,000 consecutive patients referred to the laboratory prior to training and 500 consecutive patients referred to the laboratory approximately one month after training. Thick blood smears were stained with $10 \%$ Giemsa for 10 minutes and read by health facility staff as part of their usual practice. Thick blood smears were fixed and subsequently sent to a central laboratory in Kampala, Uganda for reading by expert microscopists who were blinded to field results. Asexual parasitemia of any level was reported as a positive smear, and smears were considered negative if the examination of 100 high-power fields did not show asexual parasites. When results of the field reading and the first expert reading were discrepant, smears were reviewed by a second expert microscopist. Results were considered final if the first and second expert readings agreed. If the first and second expert readings were discordant the tie was broken by a third expert reader. 
Data management and statistical analysis. Individual patient-level surveillance data were entered into a centralized database using Access (Microsoft Corporation, Redmond, WA). Statistical analysis was done using Stata version 10 (Stata, College Station, TX). For analysis of microscopy data at individual sites, the sensitivity and specificity of field microscopy (with expert microscopy as the gold standard) for pre-training $(\mathrm{n}=1,000)$ and post-training $(\mathrm{n}=500)$ blood smears were compared using the chi-square test. We compared surveillance data collected 120 calendar days prior to the last day of training with data collected 120 calendar days after the last day of training for the following four outcome measures: 1) proportion of patients suspected of having malaria who were referred for microscopy; patients suspected of having malaria were defined as any patient sent for microscopy or any patient not sent for microcopy and given a clinical diagnosis of malaria; 2) proportion of patients with a positive blood smear who were prescribed antimalarial treatment; 3 ) proportion of patients with a negative blood smear who were prescribed antimalarial treatment; and 4) proportion of patients prescribed antimalarial treatment whose prescription included an appropriate regimen. An appropriate regimen was defined as the government's recommended first-line treatment, artemether-lumefantrine, or the second-line treatment, quinine.

Two analyses were conducted for each of the outcome variables: 1) analysis of individual sites, and 2) summary analysis of all sites in which the site was the unit of analysis. For analysis of individual sites, the unit of analysis was one working day rather than the patient. Surveillance data were not available on the name of the patient or the provider. Consequently, it was not possible to perform the analysis with hierarchical models that adjust for potential repeated observations of the same patient and same clinician. In particular, the providers at a facility on a given day may have had a practice style that was similar across patients leading to autocorrelation. To adjust for autocorrelation with available information, data were aggregated and weighted by the number of patients seen each day using survey estimation to compute means and the Wald test for hypothesis testing of pre-training and posttraining comparisons. The issue of autocorrelation did not arise in the summary analysis with the site as the unit of analysis. A single proportion was computed for an outcome for all of the patients pre-training and a second proportion for all of the patients post-training at each site. The eight pretraining proportions were then compared with the eight posttraining proportions using the paired sample $t$-test. All analyses were stratified a priori for patients $<5$ years of age and patients $\geq 5$ years of age. A $P$ value $<0.05$ was considered statistically significant.

\section{RESULTS}

Study sites. This analysis includes surveillance data from the 8 sites for periods of 120 days before and after training. Table 1 shows baseline characteristics of the sites, including the range of transmission intensity, number of patients seen, and proportion of blood smears positive. These sites vary widely in malaria endemicity, ranging from Kabale, a highland area with low transmission to three sites where transmission intensity is holoendemic, and the entomologic inoculation rates have been estimated to be more than one infective bite per person per day. ${ }^{17,18}$ Prior to training the average number of patients seen per working day ranged from 55 to 114 across the 8 sites. After training, the average number of patients seen per working day was similar, except in Kabale and Kamwezi, where the number of patients seen increased significantly. The proportion of patients $<5$ years of age ranged from $15 \%$ in the lowest transmission site to $20-33 \%$ in the higher transmission sites prior to training and did not change significantly after training. Prior to training, the proportion of blood smears read as positive ranged from $21 \%$ in the lowest transmission sites to $62 \%$ in the highest transmission site, which did not change significantly after training, except in Kabale and Kamwezi. At those sites, an indoor residual spraying program was implemented shortly after training, which is likely to have contributed to the decrease in positive blood smears.

Diagnostic accuracy of field microscopy. As shown in Table 2 , the sensitivity of field microscopy, i.e., correctly identifying the presence of malaria parasites, was relatively high before training (greater than $85 \%$ at 5 of 7 sites) and did not improve significantly after training. One site had a non-significant improvement in sensitivity from $66.7 \%$ to $85.7 \%$ and two sites had a significant decrease in sensitivity. The specificity of field microscopy, i.e., correctly reading a blood smear as negative, was also high before training (greater than $90 \%$ at 5 of 7 sites) and did not improve significantly after training (with a significant decrease in specificity at one site, Nagongera).

Proportion of patients suspected of having malaria referred for microscopy. The proportion of patients suspected of having malaria referred for microscopy increased after training at all sites, with the exception of Omugo (Figure 1). When results for individual sites were considered, the increase was statistically significant for patients $<5$ years of age at 3 of the 8 sites and for patients $\geq 5$ years of age at 5 of the 8 sites.

TABLE 2

Diagnostic accuracy of field microscopy before and after training, Uganda*

\begin{tabular}{|c|c|c|c|c|c|c|}
\hline \multirow[b]{2}{*}{ Study site $\dagger$} & \multicolumn{2}{|c|}{ Sensitivity, \% (95\% CI) } & \multirow[b]{2}{*}{$P$} & \multicolumn{2}{|c|}{ Specificity, \% $(95 \% \mathrm{CI})$} & \multirow[b]{2}{*}{$P$} \\
\hline & Pre-training & Post-training & & Pre-training & Post-training & \\
\hline Kabale & $66.7(49.8-80.9)$ & 85.7 (71.5-94.6) & 0.07 & 92.5 (90.7-94.1) & $94.0(91.4-96.0)$ & 0.32 \\
\hline Kihihi & $91.5(88.1-94.1)$ & $91.9(87.2-95.3)$ & 0.85 & $96.9(95.2-98.1)$ & $94.7(91.5-96.9)$ & 0.10 \\
\hline Walukuba & $85.2(80.4-89.2)$ & $72.7(59.0-83.9)$ & 0.03 & $81.9(78.9-84.6)$ & $84.0(80.3-87.3)$ & 0.34 \\
\hline Kyenjojo & $89.7(86.5-92.3)$ & $84.0(77.8-89.0)$ & 0.05 & $93.0(90.5-94.9)$ & $95.6(92.7-97.6)$ & 0.11 \\
\hline Omugo & $93.5(90.7-95.6)$ & $94.9(88.6-98.3)$ & 0.82 & $90.9(88.2-93.1)$ & $88.7(85.1-91.7)$ & 0.27 \\
\hline Nagongera & $79.1(74.6-83.0)$ & $85.4(70.8-94.4)$ & 0.34 & $90.0(87.3-92.2)$ & $78.8(74.8-82.5)$ & $<0.001$ \\
\hline Aduku & $97.7(96.3-98.6)$ & $88.2(82.6-92.4)$ & $<0.001$ & $83.2(77.7-87.8)$ & $88.9(84.8-92.1)$ & 0.06 \\
\hline
\end{tabular}

$* \mathrm{CI}=$ confidence interval.

$\dagger$ Data are not available for the eighth site (Kamwezi) 

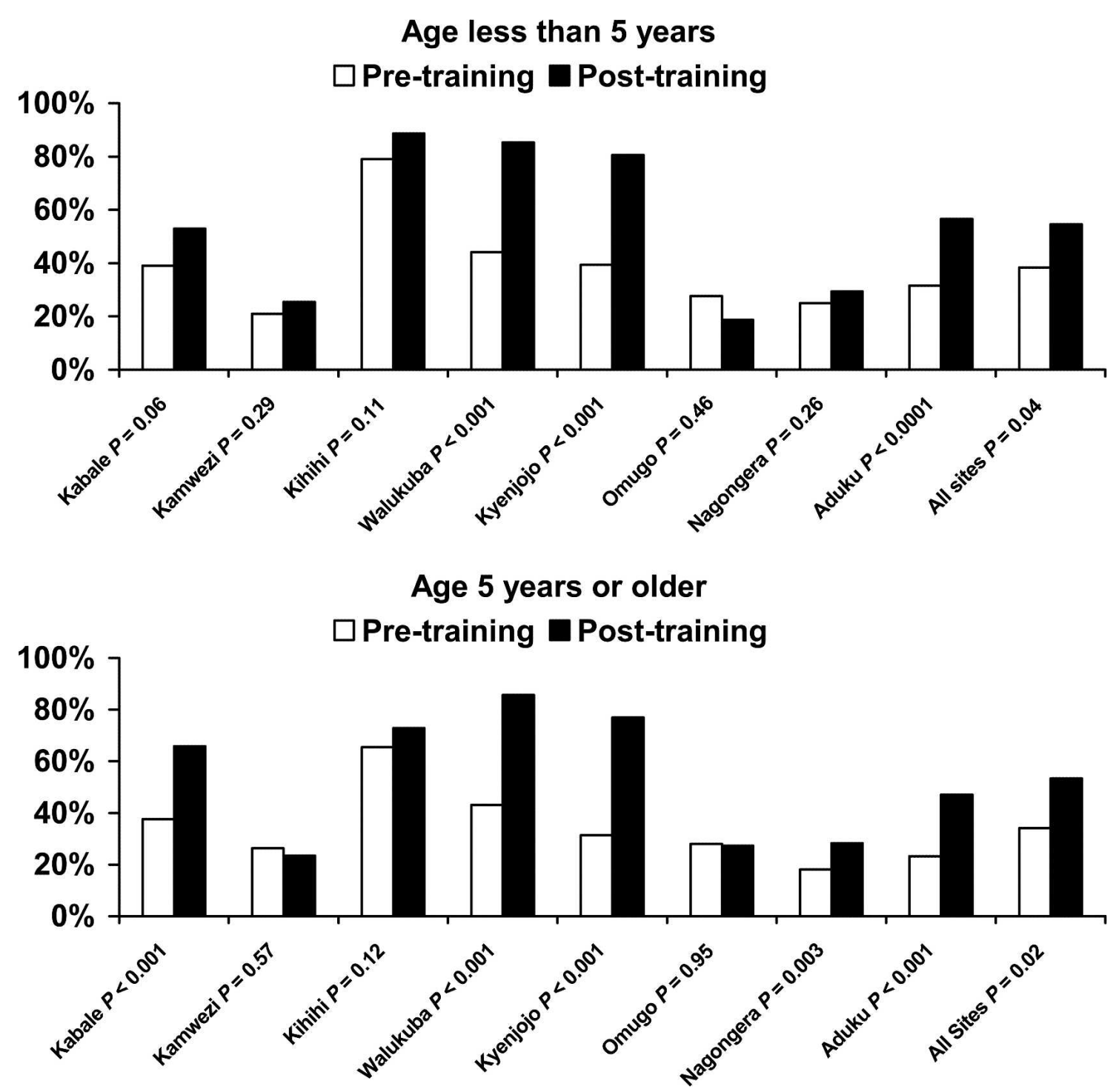

FIGURE 1. Proportion of patients suspected of having malaria who were referred for microscopy, stratified by site and age group.

Pre-training, Kihihi was the only site where more than $50 \%$ of patients suspected to have malaria in both age groups were sent to the laboratory; post-training, this benchmark was met at 5 of the sites for patients $<5$ years of age and at 4 of the sites for patients $\geq 5$ years of age. With the site as the unit of analysis, the proportion increased from $38.3 \%$ pre-training to $54.6 \%$ post-training for patients $<5$ years of age $(P=0.04)$ and from $34.1 \%$ to $53.4 \%$ for patients $\geq 5$ years of age $(P=$ 0.02).

Treatment practices. Most patients with a positive blood smear were prescribed antimalarial drugs at all the sites prior to training (range for patients $<5$ years of age $=92.1-97.8 \%$; range for patients $\geq 5$ years of age $=83.3-95.3 \%$ ), and no significant changes were seen after training (Figure 2). With the site as the unit of analysis, the proportion increased from $95.0 \%$ pre-training to $96.7 \%$ post-training for patients $<5$ years of age $(P=0.06)$ and from $90.4 \%$ to $90.8 \%$ for patients $\geq 5$ years of age $(P=0.87)$. A relatively high proportion of patients with negative blood smears were prescribed antimalarial drugs at all of the sites prior to training (range for patients $<5$ years of age $=30.7-77.7 \%$; range for patients $\geq 5$ years of age $=26.6-68.8 \%$ ). After training, the proportion of patients with negative smears who were prescribed antimalarial treatment decreased at all sites, for both age groups (Figure 3). When results for individual sites were considered, the decrease was statistically significant for patients $<5$ years of age at 6 of the 8 sites and for patients $\geq 5$ years of age at 7 of the 8 sites. With the site as the unit of analysis, the proportion decreased from $47.9 \%$ pre-training to $19.6 \%$ post-training for patients $<5$ years of age $(P<0.001)$ and from $38.8 \%$ to $15.6 \%$ for patients $\geq 5$ years of age $(P<0.001)$.

Prior to training, the proportion of patients prescribed antimalarial drug treatment whose prescription included an appropriate regimen ranged widely from $73.1 \%$ to $96.6 \%$ in patients $<5$ years of age and from $40.0 \%$ to $98.7 \%$ in patients $\geq 5$ years of age. After training, results were mixed across the sites with statistically significant improvements at 2 of the 8 sites in patients $<5$ years of age and at 4 of the 8 sites in patients $\geq 5$ years of age (Figure 4 ). The remainder of the comparisons were not significant, with the exception of Aduku where there was a significant decrease in the proportion of patients prescribed appropriate therapy. With the site 


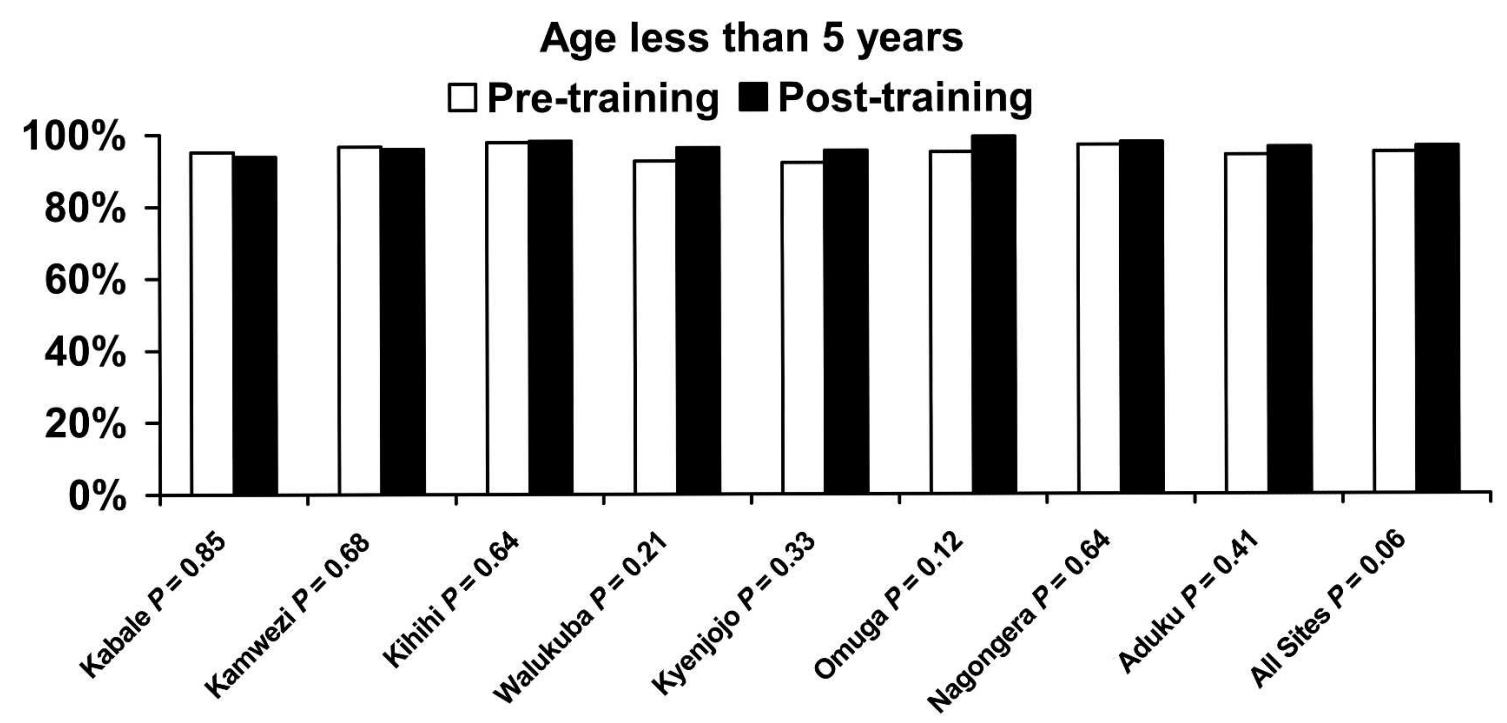

Age 5 years or older

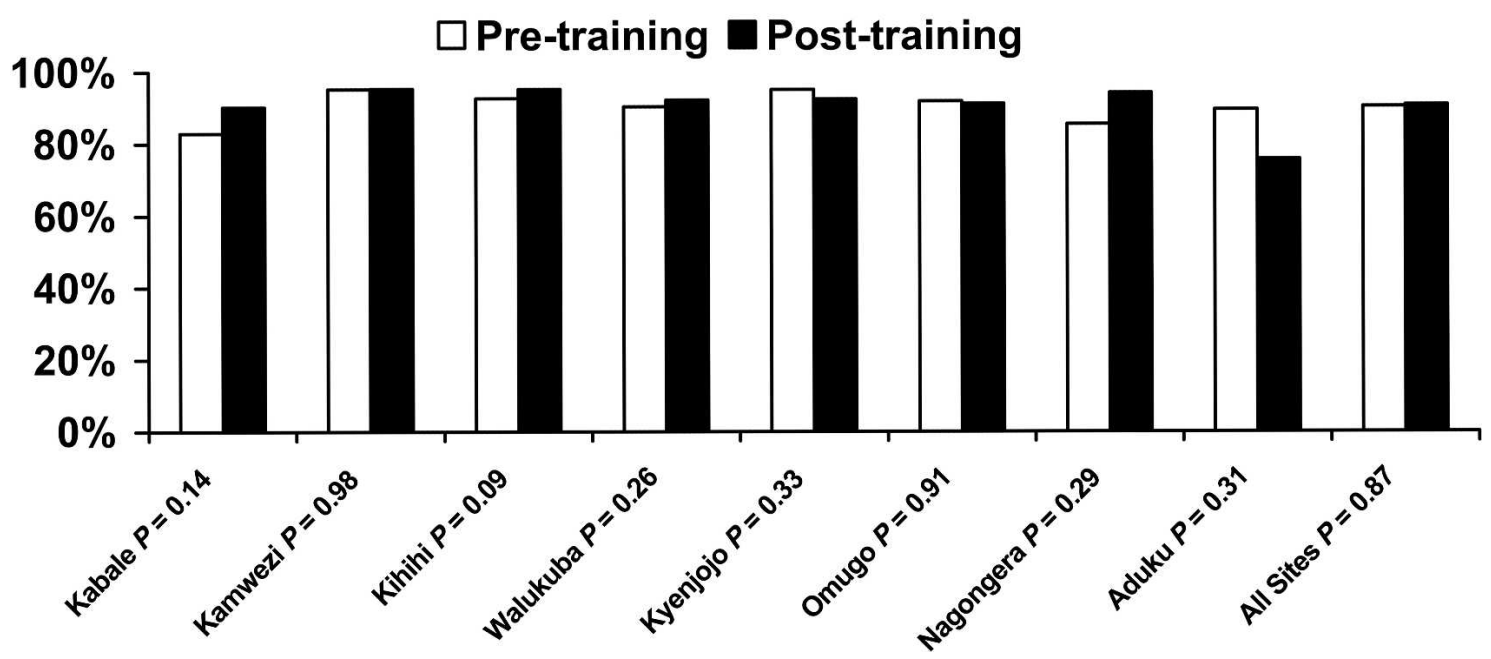

FiguRE 2. Proportion of patients with a positive blood smear who were prescribed antimalarial treatment, stratified by site and age group.

as the unit of analysis, the proportion of patients prescribed antimalarial treatment whose prescription included an appropriate regimen increased from $86.3 \%$ pre-training to $89.0 \%$ post-training $(P=0.39)$ for patients $<5$ years of age and from $78.6 \%$ to $79.5 \%$ for patients $\geq 5$ years of age $(P=0.86)$.

\section{DISCUSSION}

With the widespread implementation of ACTs in Africa, there is a critical need for improved malaria case management, ${ }^{19}$ with a greater emphasis on treatment based on laboratory confirmation. ${ }^{20}$ Several studies from Africa have reported that less than $50 \%$ of patients suspected of having malaria undergo diagnostic testing even when these services are available..$^{3,11,21}$ Another common practice is the failure to use the results of diagnostic testing rationally when making treatment decisions. Studies across a wide range of epidemiologic settings from Kenya, Tanzania, and Zambia have documented that $35-79 \%$ of patients with a negative diagnostic test result were still prescribed antimalarial drug therapy..$^{3,4,21,22}$ Improved training of health workers in Africa will be essential to promote the rational use of ACTs and extend the lifespan of these highly effective drugs.

To the best of our knowledge, there are relatively few published studies evaluating the impact of training on malaria case management. A prospective study in Guinea-Bissau found reduced mortality after a training and implementation of standardized guidelines for malaria management in a hospital setting. ${ }^{23}$ A survey study of the impact of IMCI training in Uganda noted significant associations between training and health workers' demonstrated ability to correctly classify children's illnesses, to provide correct treatment of those requiring an antibiotic or antimalarial, and to advise caregivers effectively. ${ }^{24}$ Other studies that have considered training as a factor in quality of case management illustrate the challenges in altering health workers' diagnostic and prescribing practices. In Tanzania, malaria rapid diagnostic tests were introduced with a basic training course, but this intervention did not lead to a reduction in overuse of antimalarial drugs. ${ }^{5}$ However, a retrospective assessment found a significant de- 

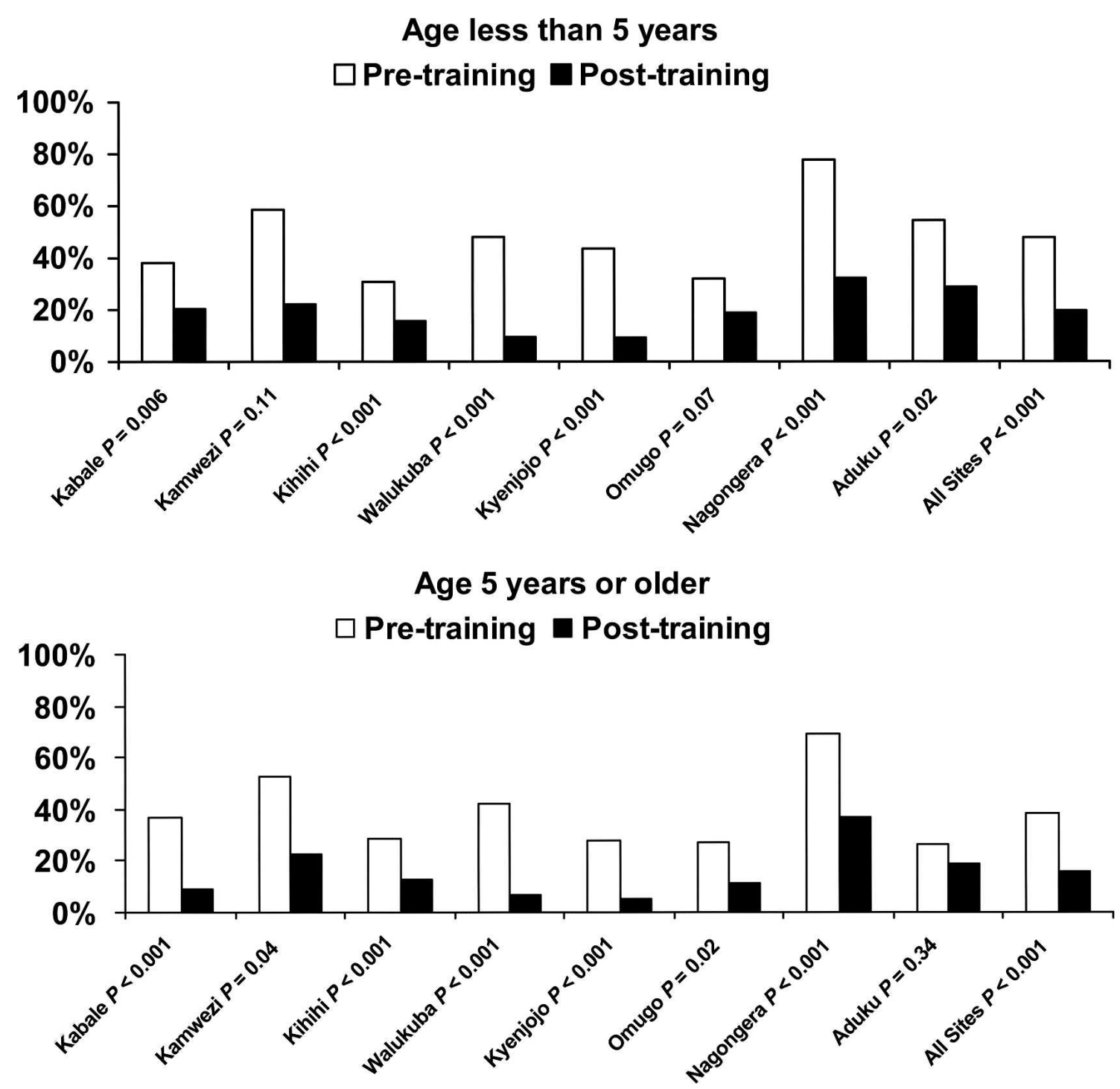

FIGURE 3. Proportion of patients with a negative blood smear who were prescribed antimalarial treatment, stratified by site and age group.

crease in malaria over-diagnosis associated with a sustained training and supportive supervision effort. ${ }^{16}$ Pariyo and others also highlighted the importance of follow-up visits, finding that the quality of care was higher at facilities with at least one supervision visit every six months compared with other facilities. ${ }^{24}$ In summary, earlier studies demonstrate that case management training may lead to improvements in health care, although challenges remain in optimizing the impact of such training efforts.

The results of this study confirm the potential for training to effect significant improvements in some areas of malaria case management across sites that represented a wide range of malaria transmission intensities. Particularly encouraging are the increase in the proportion of patients suspected of having malaria referred for laboratory confirmation, and the reduction in antimalarial drug treatments prescribed to patients with negative blood smears. After training, the proportion of patients suspected of having malaria referred for microscopy increased by approximately $50 \%$, and the proportion of patients with a negative blood smear prescribed antimalarial drug therapy decreased by approximately $60 \%$.
When the data from all 8 health facilities combined were extrapolated, these improvements resulted in 8,151 fewer prescriptions for antimalarial drug therapy among the 67,705 patients over the 120 days after training. The training program did not have a significant impact on the proportion of patients with positive blood smears who were prescribed antimalarial drug therapy. However, the pre-training standard for this indicator was already high, with more than $90 \%$ of patients with a positive blood smear prescribed antimalarial drug therapy.

The training program was less successful in improving the proportion of patients prescribed appropriate antimalarial drug therapy and the diagnostic accuracy of field microscopy. Prior to training the proportion of patients whose prescription included an appropriate antimalarial drug regimen was $86 \%$ in children $<5$ years of age and $79 \%$ in those $\geq 5$ years of age. Most appropriate prescriptions were for the government's newly recommended first-line treatment, artemetherlumefantrine $(83 \%)$, and the remaining appropriate prescriptions were for quinine, the government's recommended second-line treatment. These results were better than those in a recent study from Zambia in which $27 \%$ of children weighing 


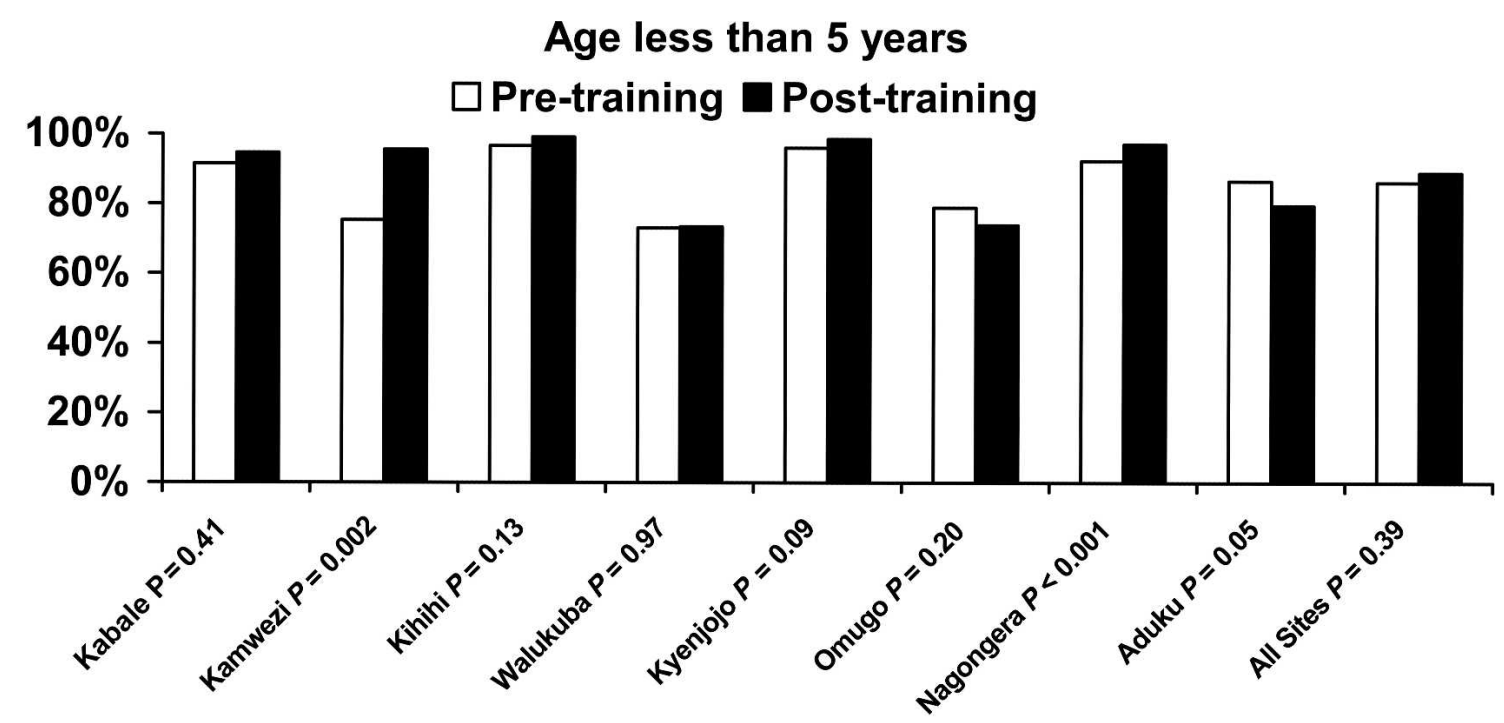

Age 5 years or older

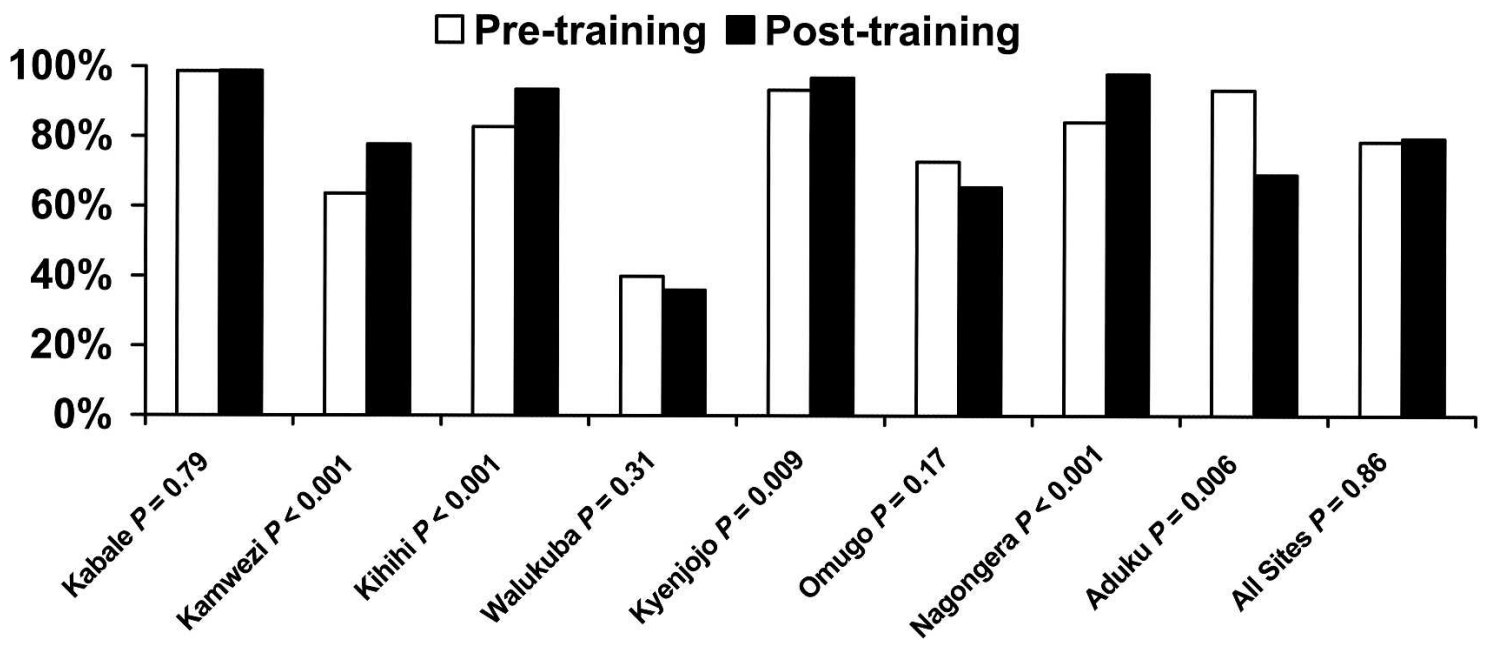

FIGURE 4. Proportion of patients prescribed antimalarial treatment whose prescription included an appropriate regimen, stratified by site and age group.

5-9 $\mathrm{kg}$ and $42 \%$ of children weighing $\geq 10 \mathrm{~kg}$ with uncomplicated malaria were treated with artemether-lumefantrine. The Zambian study was conducted two years after the facilities began the process of implementing this drug as the recommended first-line therapy. ${ }^{15}$ In our study, most patients not given appropriate therapy were prescribed chloroquine plus sulfadoxine-pyrimethamine (87\%), the previous first-line treatment of uncomplicated malaria in Uganda. Although data were not collected systematically about case management decisions, health workers reported that the primary reason for not prescribing appropriate therapy was that supplies of artemether-lumefantrine were depleted, which occurred variably from site to site both before and after the training period. For example, Aduku experienced a long period of depletion of artemether-lumefantrine after the training, which could explain the significant decrease in appropriate antimalarial drug therapy at that site.

Failure to improve the diagnostic accuracy of field microscopy after training was also felt to be caused by a combination of surprisingly good pre-training performance and limi- tations extraneous to the training intervention. Our overall sensitivity and specificity prior to training of $86 \%$ and $90 \%$, respectively, was considerably higher than reported accuracy of field microscopy from other studies in Africa. ${ }^{4,25}$

There were several strengths and limitations in this study. This study is among the first to use an independent facilitybased surveillance system to evaluate the effectiveness of a training program on key indicators of malaria case management. Surveillance data have the advantage of providing ongoing information about the quality of care and are likely to be more representative of actual performance compared with direct observation of provider-patient interactions. However, our pre-test/post-test study design may have been biased because of a Hawthorne effect, whereby health worker performance may have been improved by increased attention and not by the training program itself. Our integrated training approach was designed to involve all aspects of patient management, including diagnosis and treatment of non-malaria illnesses. By using team-based training, we attempted to emphasize the importance of all staff members of the health care 
facility working together. However, our six-day training program with supervised follow-up visits was labor-intensive and required considerable resources.

In summary, results of this study demonstrate that a relatively brief integrated training program, with supportive follow-up supervision, can achieve a significant impact on the quality of malaria case management. Improved use of laboratory confirmation of malaria cases and reduced prescription of antimalarial drugs for patients with negative blood smears are critical for rational use of antimalarial drugs and effective patient care. For optimal impact, integrated training must be accompanied by investment in other components of the health care system, including adequate staffing and reliable supplies of drugs and laboratory materials.

Received August 9, 2008. Accepted for publication September 18, 2008.

Acknowledgments: We thank the staff of participating health facilities for their enthusiastic cooperation with the training intervention evaluated in this study; the Uganda Malaria Control Program and other stakeholders who participated in development and approval of the training program; and the trainers, the JUMP team, and the UMSP data management team for their dedication and effort. This report is dedicated to Dr. Merle A. Sande (1939-2007), whose vision led to the creation of the Infectious Diseases Institute as an academic center of excellence at Makerere University and whose generous spirit brought together colleagues from several academic institutions and research projects to create the Joint Uganda Malaria Training Program.

Financial support: The study was supported by the Exxon Mobil Corporation, the Centers for Disease Control and Prevention (U50/ CCU925122), and the National Institutes of Health (AI065457-01). Grant Dorsey is a recipient of the Doris Duke Charitable Foundation Clinical Scientist Development Award.

Authors' addresses: Umaru Ssekabira and Allen Namagembe, Joint Malaria Training Program, c/o Infectious Diseases Institute, Mulago Hospital Complex, PO Box 22418, Kampala, Uganda, E-mails: ussekabira@idi.co.ug and anamagembe@idi.co.ug. Hasifa Bukirwa, Adoke Yeka, Moses Kiggundu, and Fred Wabwire-Mangen, Uganda Malaria Surveillance Program, c/o MUUCSF Malaria Research Collaboration, PO Box 7475, Kampala, Uganda, E-mails: hbukirwa@ muucsf.org, yadoke@muucsf.org, mosesk_33@hotmail.com, and fwabwire@musph.ac.ug. Heidi Hopkins, University of California, San Francisco, c/o MUUCSF Malaria Research Collaboration, PO Box 7475, Kampala, Uganda, E-mail: hhopkins@medsfgh.ucsf.edu. Marcia R. Weaver, International Training and Education Center on HIV (I-TECH) University of Washington, 901 Boren, Suite 1100, Seattle, WA 98104, E-mail: mweaver@u.washington.edu. Lydia Mpanga Sebuyira, Infectious Diseases Institute, Mulago Hospital Complex, PO Box 22418, Kampala, Uganda, E-mail: Impangasebuyira@idi.co.ug. Linda Quick, 2190 Kampala Place, Kampala, Uganda, E-mail: maq2@ug.cdc.gov. Sarah Staedke, London School of Hygiene and Tropical Medicine, c/o MUUCSF Malaria Research Collaboration, PO Box 7475, Kampala, Uganda, E-mail: sarah.staedke@lshtm.ac.uk. Gisela Schneider, DIFAEM, Paul Lechler Strasse 24, D 72076, Tubingen, Germany, E-mail: schneider@difaem.de. Keith McAdam, London School of Hygiene and Tropical Medicine, Keppel Street London WC1E 7HT, United Kingdom, E-mail: Keith.McAdam@lshtm.ac.uk. Grant Dorsey, University of California, San Francisco, Box 0811, San Francisco, CA 94143, E-mail: gdorsey@medsfgh.ucsf.edu.

\section{REFERENCES}

1. McCombie SC, 1996. Treatment seeking for malaria: a review of recent research. Soc Sci Med 43: 933-945.

2. Ndyomugyenyi R, Magnussen P, Clarke S, 2007. Malaria treatment-seeking behaviour and drug prescription practices in an area of low transmission in Uganda: implications for prevention and control. Trans R Soc Trop Med Hyg 101: 209-215.

3. Hamer DH, Ndhlovu M, Zurovac D, Fox M, Yeboah-Antwi K, Chanda P, Sipilinyambe N, Simon JL, Snow RW, 2007. Im- proved diagnostic testing and malaria treatment practices in Zambia. JAMA 297: 2227-2231.

4. Zurovac D, Midia B, Ochola SA, English M, Snow RW, 2006. Microscopy and outpatient malaria case management among older children and adults in Kenya. Trop Med Int Health 11: 432-440.

5. Reyburn H, Mbakilwa H, Mwangi R, Mwerinde O, Olomi R, Drakeley C, Whitty CJ, 2007. Rapid diagnostic tests compared with malaria microscopy for guiding outpatient treatment of febrile illness in Tanzania: randomised trial. BMJ 334: 403.

6. Osterholt DM, Rowe AK, Hamel MJ, Flanders WD, Mkandala C, Marum LH, Kaimila N, 2006. Predictors of treatment error for children with uncomplicated malaria seen as outpatients in Blantyre district, Malawi. Trop Med Int Health 11: 1147-1156.

7. Chandramohan D, Jaffar S, Greenwood B, 2002. Use of clinical algorithms for diagnosing malaria. Trop Med Int Health 7: 45-52.

8. Olivar M, Develoux M, Chegou Abari A, Loutan L, 1991. Presumptive diagnosis of malaria results in a significant risk of mistreatment of children in urban Sahel. Trans $R$ Soc Trop Med Hyg 85: 729-730.

9. Sowunmi A, Akindele JA, 1993. Presumptive diagnosis of malaria in infants in an endemic area. Trans $R$ Soc Trop Med Hyg 87: 422.

10. Gwer S, Newton CR, Berkley JA, 2007. Over-diagnosis and comorbidity of severe malaria in African children: a guide for clinicians. Am J Trop Med Hyg 77: 6-13.

11. Amexo M, Tolhurst R, Barnish G, Bates I, 2004. Malaria misdiagnosis: effects on the poor and vulnerable. Lancet 364: 1896-1898.

12. Bell D, Wongsrichanalai C, Barnwell JW, 2006. Ensuring quality and access for malaria diagnosis: how can it be achieved? Nat Rev Microbiol 4: S7-S20.

13. Eriksen J, Tomson G, Mujinja P, Warsame MY, Jahn A, Gustafsson LL, 2007. Assessing health worker performance in malaria case management of underfives at health facilities in a rural Tanzanian district. Trop Med Int Health 12: 52-61.

14. Gerstl S, Cohuet S, Edoh K, Brasher C, Lesage A, Guthmann JP, Checchi F, 2007. Community coverage of an antimalarial combination of artesunate and amodiaquine in Makamba Province, Burundi, nine months after its introduction. Malar J 6: 94.

15. Zurovac D, Ndhlovu M, Sipilanyambe N, Chanda P, Hamer DH, Simon JL, Snow RW, 2007. Paediatric malaria casemanagement with artemether-lumefantrine in Zambia: a repeat cross-sectional study. Malar J 6: 31 .

16. Masika PM, Semarundu WJ, Urassa R, Mosha J, Chandramohan $\mathrm{D}$, Gosling RD, 2006. Over-diagnosis of malaria is not a lost cause. Malar J 5: 120.

17. Lindblade KA, Walker ED, Onapa AW, Katungu J, Wilson ML, 2000. Land use change alters malaria transmission parameters by modifying temperature in a highland area of Uganda. Trop Med Int Health 5: 263-274.

18. Okello PE, van Bortel W, Byaruhanga AM, Correwyn A, Roelants P, Talisuna A, D'Alessandro U, Coosemans M, 2006. Variation in malaria transmission intensity in seven sites throughout Uganda. Am J Trop Med Hyg 75: 219-225.

19. Breman JG, Holloway CN, 2007. Malaria surveillance counts. Am J Trop Med Hyg 77: 36-47.

20. World Health Organization, 2006. WHO Guidelines for the Treatment of Malaria. Geneva: World Health Organization. Technical document WHO/HTM/MAL/2006.1108.

21. Barat L, Chipipa J, Kolczak M, Sukwa T, 1999. Does the availability of blood slide microscopy for malaria at health centers improve the management of persons with fever in Zambia? Am J Trop Med Hyg 60: 1024-1030.

22. Reyburn H, Ruanda J, Mwerinde O, Drakeley C, 2006. The contribution of microscopy to targeting antimalarial treatment in a low transmission area of Tanzania. Malar J 5: 4.

23. Biai S, Rodrigues A, Gomes M, Ribeiro I, Sodemann M, Alves F, Aaby P, 2007. Reduced in-hospital mortality after improved management of children under 5 years admitted to hospital with malaria: randomised trial. $B M J$ 335: 862.

24. Pariyo GW, Gouws E, Bryce J, Burnham G, 2005. Improving facility-based care for sick children in Uganda: training is not enough. Health Policy Plan 20 (Suppl 1): i58-i68.

25. El-Nageh MM, 1996. Coordination for better laboratory services. World Health Forum 17: 200-202. 